\title{
In vivo endomicroscopy of donor duodenum improves early detection of pancreas rejection in a recipient of simultaneous duodenum-drained pancreas-kidney transplantation: a case report
}

\author{
Krystian Żuk1, Marek Durlik2,3, Andrzej Rydzewski33,4, Grażyna Rydzewska1,3 \\ ${ }^{1}$ Department of Gastroenterology, Central Clinical Hospital of the Ministry of Internal Affairs, Warsaw, Poland \\ 2Department of Gastroenterology and Transplant Surgery, Central Clinical Hospital of the Ministry of the Internal Affairs, Warsaw, \\ Poland \\ ${ }^{3}$ Department of Surgical Research and Transplantology, Mossakowski Medical Research Centre, Polish Academy of Sciences, Warsaw, \\ Poland \\ ${ }^{4}$ Department of Nephrology, Central Clinical Hospital of the Ministry of Internal Affairs, Warsaw, Poland
}

Videosurgery Miniinv 2013; 8 (4): 366-368 DOI: 10.5114/wiitm.2013.39836

\begin{abstract}
Confocal laser endomicroscopy (CLE) is a new technique, which allows subsurface histological diagnosis at a cellular and subcellular level in vivo and could provide histological diagnosis during endoscopic examination. Up to now histological examination of the tissue sample is the only definitive way of diagnosis and monitoring organ rejection after transplantation. In case of pancreas transplantation percutaneous pancreas biopsy under imaging control is still the method of choice for obtaining tissue samples. However in 73\&\#8211;89\% of biopsy attempts, it was shown that the duodenal histology predicts the initial diagnosis of rejection of the pancreas, usefulness of transplanted duodenum biopsies for graft rejection monitoring was also described. The histology technique is time consuming, and the therapeutic decision could not be made quickly, in spite of clinical necessity. In this paper we described feasibility of visualization and biopsy of donor duodenum and detection of microscopic changes in 2 cases of transplanted duodenum, expressed as destruction of the villi and dispersed goblet cells in comparison to a microscopic view of their own healthy duodenum No or only small, endoscopically non-significant macroscopic changes in transplanted duodenum in those patients were observed. In both cases, the histological examination confirmed acute organ rejection. We demonstrated for the first time that CLE is promising and effective method to detect acute phase of organ rejection and also for follow up in those patients.
\end{abstract}

Key words: endomicroscopy, pancreas transplantation, pancreas rejection.

\section{Introduction}

Endoscopy of the digestive tract has been evolving from primary diagnostic to extensive diagnostic and therapeutic modalities with a great impact on the management of gastrointestinal pathologies [1-3]. Novel imaging technologies including virtual colonoscopy or computed tomography/magnetic reso- 

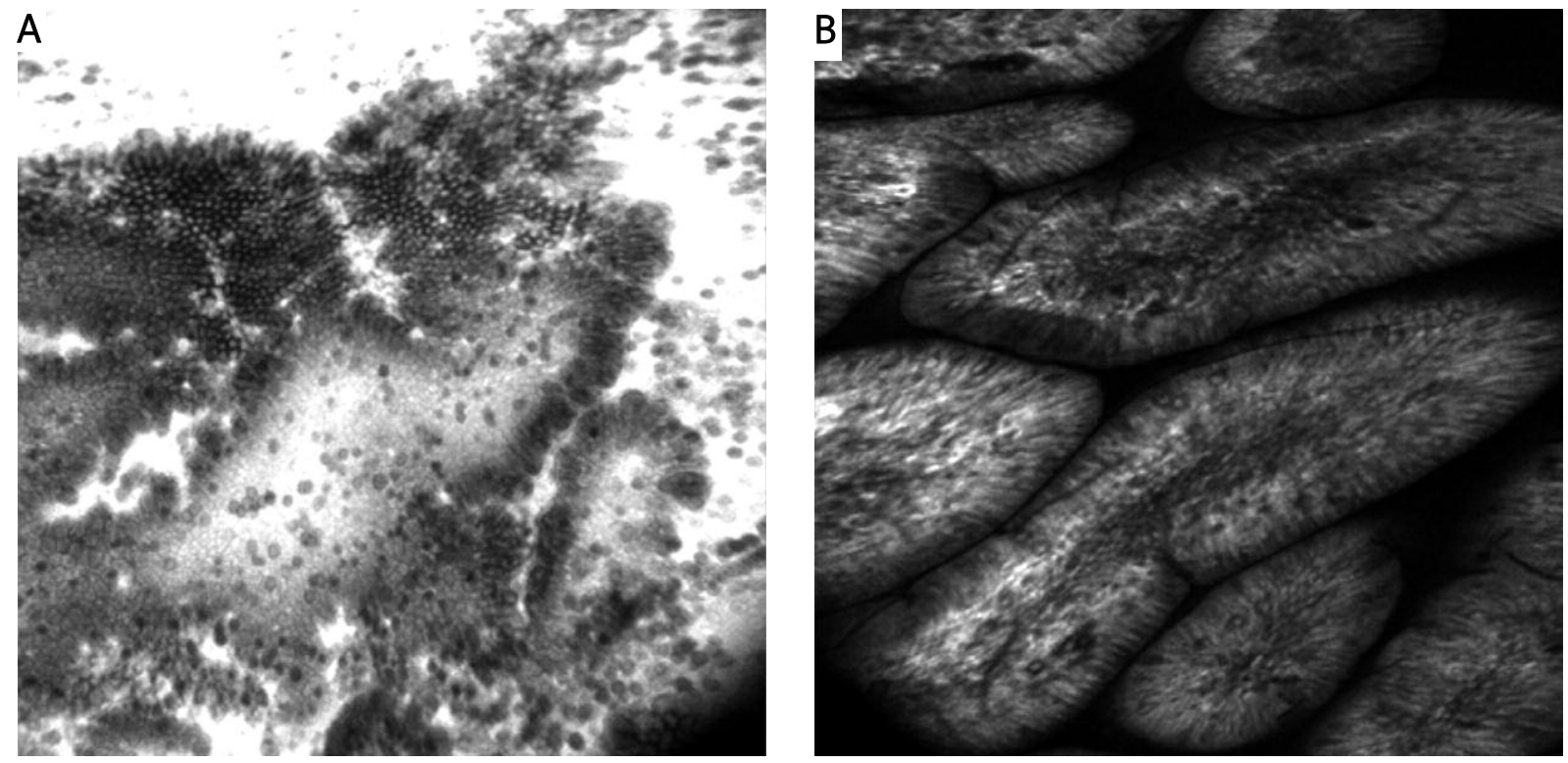

Photo 1. Endomicroscopic picture of transplanted duodenum (A - clinical and histopathological rejection, $\mathrm{B}$ - normal picture of transplanted duodenum)

nance (CT/MR) colonography, CT or magnetic resonance imaging (MRI) enterography and capsule endoscopy have also been developed and are of clinical importance [4, 5]. Minimally invasive therapeutic methods including natural orifice translumenal endoscopic surgery cholecystectomy (NOTES) [6], or endoscopic retrograde cholangiopancreatography (ERCP) in resolution of bile duct obstruction [7] are also safe and effective for selected patients. Magnifying endo-scopy with different chromoendoscopy techniques and novel endoscopic imaging as confocal endomicroscopy has become essential for determining and monitoring different pathologies in the digestive tract $[1,8]$.

Confocal laser endomicroscopy (CLE) is a new, powerful technique, which allows subsurface histological diagnosis at a cellular and subcellular level in vivo, and could provide histological diagnosis in vivo during endoscopic examination [1, 2]. This technology is widely investigated in different gastrointestinal diseases and becomes an alternative to conventional histology. The power of endomicroscopy in screening and surveillance colonoscopy, ulcerative colitis, Barrett's esophagus, and gastric cancer have been demonstrated in recent studies [1, 8]. For some gastrointestinal malignancies, a rapid in vivo diagnosis of early cancer or premalignant lesions is highly important for successful therapy. The endomicroscopy is a safe procedure, complications are rare, comparable to conventional endoscopy.
Up to now histologic examination of the tissue sample is the only definitive way of diagnosing and monitoring pancreatic rejection. Percutaneous pancreas biopsy under imaging control is still the method of choice for obtaining tissue samples. Adequate samples are obtained in this invasive method, however, in $73-89 \%$ of biopsy attempts, it was shown that the duodenal histology predicts the initial diagnosis of rejection of the pancreas, being less accurate for monitoring recovery from rejection. It was also reported that the rejection process might occur independently in either organ [8-10]. We described also usefulness of transplanted duodenum biopsies for graft rejection monitoring [11]. However, the histology technique is time consuming, and the therapeutic decision could not be made quickly, in spite of clinical necessity.

\section{Case report}

Therefore, an endomicroscopic approach to detect in vivo histological picture of transplanted duodenum might be of clinical importance in detection and monitoring of acute pancreas rejection, and its influence on a potential clinical decision.

During the first 2 weeks after simultaneous duodenal-drained pancreas-kidney transplantation (SPK) procedure for type 1 diabetes mellitus, 18 patients were examined with CLE technique. No difficulties to 
reach anastomosis with CLE were observed. Endoscopy was technically simple and not time consuming. Histological biopsies from transplanted and own duodenum were also taken for confirmation of in vivo observations. There were no complications associated with endoscopy, or downloading material for histological examination. All procedures were performed under general anesthesia.

In the group of 18 patients after SPK transplantation, we found in 2 cases microscopic changes in transplanted duodenum, expressed as destruction of the villi and dispersed goblet cells in comparison to a microscopic view of their own healthy duodenum (Photo 1). No or only small, endoscopically non-significant macroscopic changes in transplanted duodenum in those patients were observed. In both cases, the histological examination confirmed acute organ rejection.

\section{Conclusions}

Confocal endomicroscopy is a developing area of gastrointestinal endoscopy with expanding clinical and research applications. In our pilot study, we demonstrated for the first time that CLE is a promising and effective method to detect acute phase graftversus-host disease and also for follow up in those patients. To our knowledge, up till now there have been no reports on the use of this technique in recipients of pancreas transplant.

The procedure is easily acceptable and safe for patients and the clinical decision based on the information obtained after examination could be made quickly, what seems to be of high importance in transplantology [11-14]. Further studies are needed to confirm clinical usefulness of this technique in monitoring of patients after SPK transplantation.

\section{References}

1. Milewski J, Żuk K, Kierzkiewicz M, Rydzewska G. Confocal laser endomicroscopy - principles, clinical practice, future trends. Prz Gastroenterol 2011; 6: 1-16.

2. Motkowska M, Romatowski J, Januszko M, et al. Endoscopic ultrasound elastography in digestive tract diseases. Prz Gas troenterol 2012; 7: 63-9.

3. Gąsiorowska A, Talar-Wojnarowska R, Łapienis M, et al. Evaluation of $\mathrm{pH}$-impedance testing in diagnosis of patients with suspected extra-oesophageal manifestations of gastroesophageal reflux disease. Prz Gastroenterol 2012; 7: 386-96.

4. Szaflarska-Popławska A, Popławski C, Mierzwa G: Utility of capsule endoscopy in children and youth - own experience. Prz Gastroenterol 2012; 7: 209-15.
5. Boduła A, Pazurek M, Woźniak B, et al. Comparison of magnetic resonance cholangiopancreatography and endoscopic retrograde cholangiopancreatography in the diagnosis of pancreatobiliary diseases. Prz Gastroenterol 2011; 6: 187-94.

6. Kobiela J, Grymek S, Wojanowska M, et al. Magnetic instrumentation and other applications of magnets in NOTES. Videosurgery Miniinv 2012; 7: 67-73.

7. Duda M, Gryga A, Czudek S, Skalický P. Twenty years of minimally invasive surgery in the Czech Republic. Videosurgery Miniinv 2011; 6: 42-7.

8. Wyszkowski M, Rydzewska G. Endoscopic surveillance in inflammatory bowel diseases. Prz Gastroenterol 2011; 6: 279-83.

9. Mark W, Hechenleitner P, Dietze O, et al. Duodenal histology for monitoring treatment of acute rejection in pancreaticoduodenal allografts in rats. Transplantation 2002; 73: 198.

10. Hawthorne WJ, Allen RD, Greenberg ML, et al. Simultaneous pancreas and kidney transplant rejection: separate or synchronous event. Transplantation 1997; 63: 352.

11. Durlik M, Kosmala W, Milewski J, et al. Feasibility of visualization and biopsy of donor duodenum by double-balloon enteroscopy technique in a recipient of simultaneous enteric-drained pancreas-kidney transplant: case report. Transplantation 2006; 82: 578-9.

12. Ćwik G, Solecki M, Ciechański A, et al. Perforations in the colon. Prz Gastroenterol 2012; 7: 125-32.

13. Baczewska-Mazurkiewicz D, Celiński K, Dłużniewski M, et al. Guidelines for the management of antiplatelet and anticoagulant therapy in patients undergoing endoscopic procedures. Recommendations of the Working Group of the Polish Society of Gastroenterology and the Polish Consultant in Gastroenterology. Prz Gastroenterol 2012; 7: 243-8.

14. Amornyotin S. Sedation-related complications in gastrointestinal endoscopy. World J Gastrointest Endosc 2013; 5: 527-33.

Received: 11.10.2013, accepted: 12.12.2013. 